\title{
Acoustic Characteristic Analysis of Prestressed Cylindrical Shells in Local Areas
}

\author{
Lu-yun Chen \\ State Key Laboratory of Ocean Engineering, Shanghai Jiaotong University, Shanghai 200240, PR China
}

Yong Liu
China Ship Scientific Research Center, Shanghai Branch, Shanghai 214082, PR China

(Received 20 July 2014; accepted 5 November 2015)

The influence of prestress on dynamic responses and acoustic radiation for thin cylindrical shells is analyzed in this study. The strain-displacement equation of cylindrical shells with prestress in local areas is established based on the Flügge theory. The structural-acoustic radiation formulation for prestressed cylindrical shells in local areas is instituted by using the variational principle. A numerical analysis is then carried out. The numerical results are validated by comparing the influence of prestress on acoustic radiation power and directivity. This study shows that prestress significantly affects the dynamic characteristics of cylindrical shells.

\section{INTRODUCTION}

Prestress (initial stress) exists in complex structures because of welding residual stress, structural manufacturing defects, material thermal effects, and static external loading. Prestress resists or aids structural deformation and alters the static and dynamic characteristics of complex structures. For example, the natural frequencies of a structure increase or decrease with prestress distribution. Several studies have demonstrated the influence of prestress on structural-acoustic radiation. A previous study analyzed the structure buckling and vibration problem of composite sandwich plates with initial stress through the higher-order finite element theory. ${ }^{1}$ The influence of prestress on the vibration frequency of concrete bridges was also investigated using the prestress stiffness matrix. ${ }^{2}$ Regarding uniform Euler-Bernoulli beams under linearly varying fully tensile, the structure natural frequencies may be increased or decreased, and parameters change the forbidden frequencies of the mechanical system, considering the pre-stress force. ${ }^{3}$ The influence of temperature was also determined by specifying the arbitrary high temperature on the outer surface and the ambient temperature on the inner surface of cylindrical shells. In this case, the prestressed state was induced by thermal loading. ${ }^{4}$ The potential influence of prestress on resonance frequencies was also assessed, and the results showed that prestress depended on water depth. ${ }^{5}$ Moreover, prestress can be used as a parameter to change the natural frequencies of a mechanical system in a proposed model of the prestressed structure. ${ }^{6}$

Thin cylindrical shells are widely used in complex structures, such as aerospace, marine, mechanical, and civil constructs. Research on the vibration and acoustic radiation of cylindrical shells has been a hot topic these past few years. A non-linear finite element model based on Murnaghan thirdorder elastic theory was applied to analyze the resonance structure, and the results showed that natural frequencies increased with increasing compressive stress. ${ }^{7}$ The free-vibration characteristics of cylindrical shells were also investigated for a general class of elastic-support boundary conditions which considered depth-water pressure. ${ }^{8}$ Moreover, several studies have investigated cylindrical shell dynamic problems with prestress distribution. A previous study reported the output power flow for an infinite ring-stiffened cylindrical shell submerged in fluid induced by a cosine harmonic circumferential line force under a uniform external hydrostatic pressure field and compared the influence of depth-water pressure. ${ }^{9}$ The effect of variation in flow velocities and hydrostatic pressures on the dynamic behavior of fluid-conveying shells, as well as that of support conditions on free vibration, were further studied; a 3D method for prestress distribution was also established. ${ }^{10,11}$ Simultaneous effects of a prestress condition, including its linear and nonlinear parts and the elastic foundation on natural frequencies of shells under various boundary conditions, were extensively examined. ${ }^{12}$ The structure dynamic response of cylindrical shells subjected to harmonic excitation at low natural frequencies was discussed by comparing five different nonlinear cylindrical shell theories. ${ }^{13}$ The free vibration and instability characteristics of a ring-stiffened cylindrical shell that conveys internal fluid was analyzed using motion equations based on the Flügge theory. The effects of fluid velocity and ring stiffener parameters on the natural frequency and stability characteristics of the shell were also assessed. ${ }^{14}$ The effect of prestress on dynamic responses of fluid and initial stresses for the pipeline were further investigated using the integral equation; the result showed that the influence of prestress at high frequencies is essential. ${ }^{15}$ The vibration problem of cylindrical shells was also evaluated using the differential quadrature method to resolve the prestressed structure problem. ${ }^{16}$

However, most studies focused on models of uniformly distributed prestress, such as hydrostatic pressure or water pressure, although fluid velocity deduces pressure. To the best of the author's knowledge, few results have investigated the prestress problem of local area distribution, despite its wide existence in engineering design and manufacturing. With this reason, to analyze the dynamic response and acoustic radiation characteristics of a prestressed complex structure in local areas is necessary. This study aimed to describe the low-frequency dynamic and acoustic responses of a prestressed cylindrical shell structure. The basic equations of structural-acoustic characteristics for cylindrical shells were established using the classical Flügge theory. The developed model was used to determine the influence of prestress on the local area. A general mathematical model capable of analyzing the dynamic behav- 


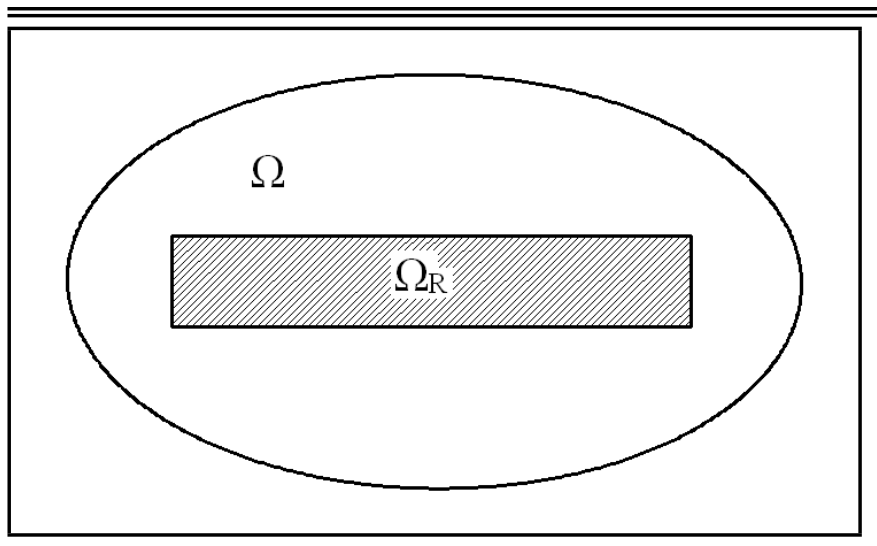

Figure 1. Prestress distribution in local areas.

ior of thin cylindrical shells with and without local area distribution as well as the overall and non-uniform distribution of prestress, were developed using the elasticity model. Finally, the effectiveness of the proposed models was confirmed through numerical calculations.

This paper is organized as follows: a brief literature review of the structural-acoustic radiation and prestress in Section 1. The basic model of prestress in local areas of a thin plate is demonstrated in Section 2. The motion governing the equations for cylindrical shells is presented in Section 3. Methods for addressing prestressed cylindrical shells in local areas in Section 4. The structural-acoustic radiation function of cylindrical shells is then established in Section 5. Acoustic radiation power and directivity are numerically analyzed in Section 6 to illustrate the validity and efficiency of the proposed method. Finally, concluding remarks are provided in Section 7.

\section{PRESTRESS MODEL}

\subsection{Prestress Model in Local Areas}

An elastic isotropic thin plate was used to establish a prestress distribution model in local areas. As shown in Fig. 1, the structure domain $\Omega$ contains the sub-domain $\Omega_{R}$ (more than one domain $\Omega_{R}$ in the domain $\Omega$ ). Prestress exists in the subdomain $\Omega_{R}$.

\subsection{Prestress Equation}

The prestress value was defined as zero in the absence of prestress in the design domain $\Omega_{R}$. The unified prestress equation of the thin plate structure was also established. A thin plate was subjected to dynamic external loading, static external loading, and initial stress. The structure stress in the design domain can be expressed as $\sigma=\left\{\sigma_{x x}, \sigma_{y y}, \sigma_{z z}, \tau_{x y}, \tau_{x z}, \tau_{y z}\right\}$. Thus, in the structure dynamic response, prestress consists of two parts: initial and static loading stresses. The initial stress consists of initial stress, welding residual stress, and material thermal effects, whereas the static loading stress is due to static external loading. Only prestress caused by static external loading was analyzed in this study.

According to the thin plate theory, the structure stress in the thin plate can be expressed as $\sigma_{z z}=\tau_{x z}=\tau_{y z}=0$, where $z$ is the direction of the plate thickness. The structure stress function can be written as $\boldsymbol{\sigma}=\left\{\sigma_{x x}, \sigma_{y y}, 0, \tau_{x y}, 0,0\right\}$. Moreover, the cylindrical polar coordinate system indicates that the structure stress is represented by $\boldsymbol{\sigma}=\left\{\sigma_{x x}, \sigma_{\theta \theta}, \tau_{x \theta}\right\}$.

According to the Flügge theory and the structure elasticity theory, structure stresses, namely $\sigma_{x x}, \sigma_{\theta \theta}$, and $\tau_{x \theta}$, are

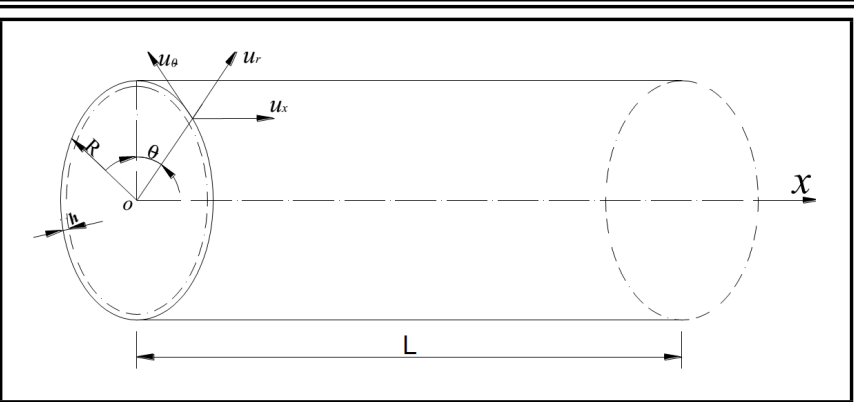

Figure 2. Coordinate system for a cylindrical shell.

related to the strain for homogeneous and isotropic materials and can be expressed as: $\sigma_{x x}=\frac{E}{1-\mu^{2}}\left(\varepsilon_{x x}+\mu \varepsilon_{\theta \theta}\right)$, $\sigma_{\theta \theta}=\frac{E}{1-\mu^{2}}\left(\varepsilon_{\theta \theta}+\mu \varepsilon_{x x}\right)$, and $\tau_{x \theta}=\frac{E}{2(1+\mu)} \gamma_{x \theta}$ respectively. The structure-strain displacements, $\varepsilon_{x x}$ and $\varepsilon_{\theta \theta}$, are in axial and circumferential directions respectively. The structure shear strain, $\gamma_{x \theta}$, is in the coordinate system, $E$ is the structural material Young's modulus, and $\mu$ is the Poisson ratio.

For isotropic materials, the structure prestress in local areas can be written as $\boldsymbol{\sigma}^{0}=\left\{\sigma_{x x}^{0}, \sigma_{y y}^{0}, \sigma_{z z}^{0}, \tau_{x y}^{0}, \tau_{x z}^{0}, \tau_{y z}^{0}\right\}$. If $\sigma^{0}=0$, then prestress does not exist in the structure design domain. Prestress has no influence on the shear strain in the thin plate. Only two principal directions of prestress are considered, and the stress can be stated as $\sigma_{z z}^{0}=\tau_{x y}^{0}=\tau_{x z}^{0}=$ $\tau_{y z}^{0}=0$. Prestress can be rewritten with a cylindrical polar coordinate system as $\boldsymbol{\sigma}^{0}=\left\{\sigma_{x x}^{0}, \sigma_{\theta \theta}^{0}\right\}$.

The cylindrical structure stress of the thin plate subjected to state loading, dynamic loading, and prestress can be expressed as $\boldsymbol{\sigma}=\boldsymbol{\sigma}^{0}+\boldsymbol{\sigma}^{f}=\left\{\sigma_{x x}^{0}, \sigma_{\theta \theta}^{0}, 0,0,0,0\right\}+$ $\left\{\sigma_{x x}^{f}, \sigma_{\theta \theta}^{f}, 0, \tau_{x \theta}^{f}, 0,0\right\}$, where $\boldsymbol{\sigma}^{0}$ is the local-area prestress, and $\boldsymbol{\sigma}^{f}$ is caused by dynamic external loading.

\section{MOTION EQUATIONS OF CYLINDRICAL SHELLS}

In this study, the dynamic response problem of prestress in the local areas of cylindrical shells was formulated in a cylindrical polar coordinate system.

\subsection{Free Vibration Formulations of Cylindrical Shells}

The structure of isotropic, infinite, and thin cylindrical shells in a fluid medium contains the region $\Omega_{S}$ with the boundary $\Gamma_{S}$. The dynamic response of the shells was determined in a cylindrical polar coordinate system $(x, \theta, r)$, as shown in Fig. 2, where the $x$-axis is the axis of the shell and $r$ and $\theta$ are the radial and circumferential directions respectively.

The general assumptions in the structural-acoustic analysis for cylindrical shells include the following: the structure material is isotropic and linearly elastic; the fluid medium is isotropic, inviscid, and incompressible; the acoustic wave equation is linear; the deformation of the shell is small; the shell and fluid gravity force are neglected; and the shell thickness $t$ is smaller than the shell's mean radius. In addition, only the low- and middle-frequency domains of acoustic radiation are discussed in this study, while structure-fluid coupling are not.

Figure 2 shows the circular cylindrical shell structure with thickness $t$, length $L$, and radius of the middle surface $R$. 
Therefore, the dynamic displacement vector $\mathbf{u}$ in the cylindrical polar coordinate system can be expressed as:

$$
\mathbf{u}=\left\{u_{x}(x, \theta, r, t), u_{\theta}(x, \theta, r, t), u_{r}(x, \theta, r, t)\right\}^{T} ;
$$

where $u_{x}, u_{\theta}$, and $u_{r}$ represent the displacements of an arbitrary point on the middle surface of shell axial, tangential, and radial displacements respectively. The superscript $T$ is the transposition of a vector/matrix in the equation. Dynamic displacement in the middle surface of the cylindrical shell structure can be obtained using the classical Flügge theory and can be expressed as:

$$
\begin{aligned}
& u_{x}=\sum_{n=-\infty}^{\infty} \int_{-\infty}^{\infty} U_{x}\left(k_{x}\right) e^{\left(i n \theta+i k_{x} x\right)} d x, \\
& u_{\theta}=\sum_{n=-\infty}^{\infty} \int_{-\infty}^{\infty} U_{\theta}\left(k_{x}\right) e^{\left(i n \theta+i k_{x} x\right)} d x,
\end{aligned}
$$

and

$$
u_{r}=\sum_{n=-\infty}^{\infty} \int_{-\infty}^{\infty} U_{r}\left(k_{x}\right) e^{\left(i n \theta+i k_{x} x\right)} d x
$$

where $U_{x}, U_{\theta}$, and $U_{r}$ are the shell spectral displacement amplitudes of the axial, tangential, and radial directions respectively. The parameter $k_{x}$ is the wave number in the axial direction.

\subsection{Strain-displacement Equation of Cylindrical Shells}

The cylindrical shell structure loaded by residual stress or static loading can resist or aid structure deformation. The strain field associated with the vibration of a prestressed cylindrical shell consists of prestress and dynamic stress. As such, the strain-displacement relations include several nonlinear terms. Therefore, the total strain field $\varepsilon$ for prestressed cylindrical shells may be represented as the cylindrical polar coordinate system using:

$$
\varepsilon=\varepsilon^{0}+\varepsilon^{f}=\left\{\varepsilon_{x x}^{0}, \varepsilon_{\theta \theta}^{0}, 0,0,0,0\right\}+\left\{\varepsilon_{x x}^{f}, \varepsilon_{\theta \theta}^{f}, 0, \gamma_{x \theta}^{f}, 0,0\right\} ;
$$

where $\varepsilon$ is the strain vector of the cylindrical shell and $\varepsilon^{0}$ is the strain vector caused by local-area prestress. Only the linear strain was discussed in this study, such as $\left\{\varepsilon_{x x}^{0}, \varepsilon_{\theta \theta}^{0}, 0,0,0,0\right\}$, where $\varepsilon_{x x}^{0}$ and $\varepsilon_{\theta \theta}^{0}$ are the axial and circumferential strains by prestress respectively.

The structure strain, $\varepsilon^{f}=\left\{\varepsilon_{x x}^{f}, \varepsilon_{\theta \theta}^{f}, 0, \gamma_{x \theta}^{f}, 0,0\right\}$, is the dynamic strain components in the cylindrical coordinate system and includes strain and shear strain, which was caused by harmonic external loading. Based on the classical Flügge theory, $\varepsilon_{x x}^{f}, \varepsilon_{\theta \theta}^{f}$, and $\gamma_{x \theta}^{f}$ are strain components in the cylindrical coordinate system.

In the middle surface of the structure, the relationship of the strain-displacement equation for the thin cylindrical shell structure can be expressed as: $\varepsilon_{x x}^{f}=\frac{\partial u_{x}}{\partial x}, \varepsilon_{\theta \theta}^{f}=$ $\frac{1}{R} \frac{\partial u_{\theta}}{\partial \theta}+\frac{u_{r}}{R}$, and $\gamma_{x \theta}^{f}=\frac{1}{R} \frac{\partial u_{x}}{\partial \theta}+\frac{\partial u_{\theta}}{\partial x}$. The straindisplacement equation can be written in vector form as: $\varepsilon^{f}=$ $\left\{u_{x, x}, \frac{1}{R}\left(u_{\theta, \theta}+u_{r}\right), 0, \frac{1}{R} u_{x, \theta}+u_{\theta, x}, 0,0\right\}^{T}$. Meanwhile, the strain-displacement equation can be rewritten in matrix form as:

$$
\varepsilon^{f}=\mathbf{Y} \boldsymbol{\zeta}
$$

where $\mathbf{Y}=\left[\begin{array}{llllll}1 & 0 & 0 & 0 & 0 & 0 \\ 0 & 1 & 0 & 0 & 0 & 0 \\ 0 & 0 & 0 & 0 & 0 & 0 \\ 0 & 0 & 0 & 1 & 1 & 0 \\ 0 & 0 & 0 & 0 & 0 & 0 \\ 0 & 0 & 0 & 0 & 0 & 0\end{array}\right]$ and $\boldsymbol{\zeta}=\left\{\zeta_{1}, \zeta_{2}, \zeta_{3}, \zeta_{4}, \zeta_{5}, \zeta_{6}\right\}^{T}$.

Furthermore, $\zeta_{1}=u_{x, x}, \zeta_{2}=\frac{1}{R}\left(u_{\theta, \theta}+u_{r}\right), \zeta_{4}=\frac{1}{R}\left(u_{x, \theta}\right)$, and $\zeta_{5}=u_{\theta, x}$.

\subsection{The Stress-Strain Equation of the Cylindrical Shell}

In the cylindrical shell structure, the general constituent stress-strain relationship between the stress and strain vectors can be obtained by using the following equation:

$$
\boldsymbol{\sigma}=\mathbf{D} \varepsilon=\boldsymbol{\sigma}^{0}+\mathbf{D} \varepsilon^{f}
$$

where $\boldsymbol{\sigma}^{0}$ is the prestress vector, viz. $\boldsymbol{\sigma}^{0}=\left\{\sigma_{x x}^{0}, \sigma_{\theta \theta}^{0}\right\}$ and $\sigma_{x x}^{0}, \sigma_{\theta \theta}^{0}$ are the normal prestress vector components in the cylindrical coordinate system, and $\mathbf{D}$ represents the shell stress-strain matrix. For the isotropic material structure, the constitutive relationship matrix $\mathbf{D}$ can be expressed as:

$$
\mathbf{D}=\frac{E}{(1+\mu)(1-2 \mu)}\left[\begin{array}{cccccc}
1-\mu & \mu & \mu & 0 & 0 & 0 \\
\mu & 1-\mu & \mu & 0 & 0 & 0 \\
\mu & \mu & 1-\mu & 0 & 0 & 0 \\
0 & 0 & 0 & 0.5-\mu & 0 & 0 \\
0 & 0 & 0 & 0 & 0.5-\mu & 0 \\
0 & 0 & 0 & 0 & 0 & 0.5-\mu
\end{array}\right]
$$

where $E$ is the Young's modulus, and $\mu$ is the Poisson ratio.

\subsection{Governing Formulation of the Cylindrical Shell}

In a small shell element with unit length, the displacement vector of the cylindrical shell $\mathbf{u}$ was governed by the motion equation. The dynamic displacement parameter of the cylindrical shell is $\mathbf{u}=\left\{u_{x}, u_{\theta}, u_{r}\right\}^{T}$. In the structure domain $\Omega_{S}$, the appropriate function for the shell domain can be expressed as:

$$
\rho_{S} \ddot{\mathbf{u}}-\nabla \cdot \boldsymbol{\sigma}=0 \text { in } \Omega_{S} ;
$$

where $\rho_{S}$ is the density of the cylindrical shell, dot represents a derivative with respect to time, and $\Omega_{S}$ is the shell solution domain.

If fluid-structural coupling is weak and can be neglected, especially for air, the influence of acoustic pressure on the structure can also be neglected, and the shell's motion equation can be proposed. If the distribution function of prestress is defined, the amplitude and frequency of external loading is also given. Combining the boundary condition and substituting Eqs. (3) and (4) into Eq. (5) yields the following equation that governs the motion of the cylindrical shell:

$$
\int_{\Omega_{S}} \delta \varepsilon^{T} \mathbf{D} \varepsilon d \Omega_{S}+\int_{\Omega_{S}} \delta \mathbf{u}^{T} \rho \ddot{\mathbf{u}} d \Omega_{S}-\int_{\Omega_{S}} \delta \mathbf{u}^{T} \mathbf{f} d \Omega_{S}=0
$$

where $\mathbf{u}$ is the cylindrical shell displacement vector, such as $\mathbf{u}=\left\{u_{x}, u_{\theta}, u_{r}\right\}^{T}, \boldsymbol{\rho}_{S}$ represents the inertia force acceleration matrix, and $\mathbf{f}$ is the vector of the shell body force.

These motion equations of shell in the acoustic medium can be rewritten in a variational formula by using the Hamilton 
principle. Hence, the following motion equation can be obtained:

$$
\begin{gathered}
\int_{\Omega_{S}} \delta\left(\varepsilon^{0}+\varepsilon^{f}\right)^{T} \mathbf{D}\left(\varepsilon^{0}+\varepsilon^{f}\right) d \Omega_{S}+\int_{\Omega_{S}} \delta \overline{\mathbf{u}}^{T} \mathbf{N}_{S}^{T} \rho_{S} \mathbf{N}_{S} \ddot{\overline{\mathbf{u}}} d \Omega_{S}- \\
\int_{\Omega_{S}} \delta \overline{\mathbf{u}}^{T} \mathbf{N}_{S}^{T} \mathbf{f} d \Omega_{S}=0
\end{gathered}
$$

where the first, second, and third parts represent strain energy variation in the structure, kinetic energy variation in the structure, and external force acting on the structure of the virtual variation in external forces respectively.

\section{SOLUTION OF MOTION EQUATION}

\subsection{Structure Discretization}

In Eq. (7), the governing motion of the cylindrical shell subjected to boundary conditions was solved by using the finite element method (FEM). The cylindrical shell was also discretized by introducing cylindrical frustums, in which the shell displacement variable $\mathbf{u}(x, \theta, r, t)$ and the variational form $\delta \mathbf{u}(x, \theta, r, t)$ can be expressed as:

$$
\begin{aligned}
\mathbf{u}(x, \theta, r, t) & =\left\{\mathbf{N}_{S} \overline{\mathbf{u}}_{x}, \mathbf{N}_{S} \overline{\mathbf{u}}_{\theta}, \mathbf{N}_{S} \overline{\mathbf{u}}_{r}\right\}^{T}=\mathbf{N} \overline{\mathbf{u}} \\
\delta \mathbf{u}(x, \theta, r, t) & =\left\{\delta\left(\mathbf{N}_{S} \overline{\mathbf{u}}_{x}\right), \delta\left(\mathbf{N}_{S} \overline{\mathbf{u}}_{\theta}\right), \delta\left(\mathbf{N}_{S} \overline{\mathbf{u}}_{r}\right)\right\}^{T} \\
& =\delta \overline{\mathbf{u}}^{T} \mathbf{N}^{T}
\end{aligned}
$$

where $\overline{\mathbf{u}}=\left\{\overline{\mathbf{u}}_{x}, \overline{\mathbf{u}}_{\theta}, \overline{\mathbf{u}}_{r}\right\}^{T}$ represents the nodal displacement vector. $\overline{\mathbf{u}}_{x}, \overline{\mathbf{u}}_{\theta}$, and $\overline{\mathbf{u}}_{r}$ are the nodal displacement in the axial, tangential, and radial directions respectively. $\mathbf{N}_{S}$ denotes the shape function matrix of the element and $\mathbf{N}$ is the assembled shape function matrix. The relationship between matrix $\mathbf{N}$ and matrix $\mathbf{N}_{S}$ can be obtained by using $\mathbf{N}=\left[\begin{array}{ccc}\mathbf{N}_{S} & 0 & 0 \\ 0 & \mathbf{N}_{S} & 0 \\ 0 & 0 & \mathbf{N}_{S}\end{array}\right]$.

Defining $d \boldsymbol{\zeta}=\mathbf{G} d \overline{\mathbf{u}}$, then there is $d \boldsymbol{\varepsilon}=d \varepsilon^{f}=$ YG $d \overline{\mathbf{u}}$. The transformation matrix $\mathbf{G}$ can be defined as $\mathbf{G}=\left[\begin{array}{cccccc}\mathbf{N}_{S, x}^{T} & 0 & 0 & \frac{\mathbf{N}_{S, x}^{T}}{R} & 0 & 0 \\ 0 & \frac{\mathbf{N}_{S, x}^{T}}{R} & 0 & \mathbf{N}_{S, x}^{T} & 0 & 0 \\ 0 & \frac{\mathbf{N}_{S, x}^{T}}{R} & 0 & 0 & 0 & 0\end{array}\right]^{T}$. The linear straindisplacement matrix $\mathbf{A}^{R}$ can be obtained by substituting the transformation matrix G into Eq. (4). In addition, the differential equations of the structural dynamic strain can be expressed as:

$$
d \boldsymbol{\varepsilon}^{f}=\mathbf{Y} \mathbf{G} d \overline{\mathbf{u}}=\mathbf{A} d \overline{\mathbf{u}}=\left[\begin{array}{ccc}
\frac{\partial \mathbf{N}_{S}}{\partial x} & 0 & 0 \\
0 & \frac{\partial \mathbf{N}_{S}}{R \partial x} & \frac{\mathbf{N}_{S}}{R} \\
0 & 0 & 0 \\
\frac{\partial \mathbf{N}_{S}}{R \partial \theta} & \frac{\partial \mathbf{N}_{S}}{\partial x} & 0 \\
0 & 0 & 0 \\
0 & 0 & 0
\end{array}\right] d \overline{\mathbf{u}}
$$

\subsection{Motion Function of the Cylindrical Shell}

Nonlinear terms (second- and third-order) were neglected under low shell deformation and only the nonlinear straindisplacement relationship were used to derive the geometric stiffness matrix during standard discretization. The following equation governing the motion of the shell in the global coordinate system was obtained using the variational principle. By substituting Eqs. (3), (8), and (9) into Eq. (7), a motion equation can be yielded, which governs the motion of the cylindrical shell system as a standard discretization form:

$$
\begin{gathered}
\int_{\Omega_{S}} \mathbf{N}_{S}^{T} \rho_{S} \mathbf{N}_{S}^{T} \ddot{\mathbf{u}} d \Omega_{S}+\int_{\Omega_{S}} \mathbf{A}^{T} \mathbf{D} \mathbf{A} \overline{\mathbf{u}} d \Omega_{S}+ \\
\int_{\Omega_{S}} \mathbf{G}^{T} \mathbf{S G} \overline{\mathbf{u}} d \Omega_{S}-\int_{\Omega_{S}} \mathbf{f} d \Omega_{S}=0 ;
\end{gathered}
$$

where $\ddot{\overline{\mathbf{u}}}$ is the shell nodal acceleration vector, $\overline{\mathbf{u}}$ is the shell nodal displacement vector, and the matrix $\mathbf{S}$ is provided by the structural prestress matrix and can be written as: $\mathbf{S}=\left[\begin{array}{ccc}\sigma_{x x}^{0} \mathbf{I}_{2} & \tau_{x \theta}^{0} \mathbf{I}_{2} & \tau_{x \gamma}^{0} \mathbf{I}_{2} \\ \tau_{x \theta}^{0} \mathbf{I}_{2} & \sigma_{\theta \theta}^{0} \mathbf{I}_{2} & \tau_{\theta \gamma}^{0} \mathbf{I}_{2} \\ \tau_{x \gamma}^{0} \mathbf{I}_{2} & \tau_{\theta \gamma}^{0} \mathbf{I}_{2} & \sigma_{\gamma \gamma}^{0} \mathbf{I}_{2}\end{array}\right]$, where $\mathbf{I}_{2}$ is a unit matrix of order 2. The local area prestress $\sigma^{0}=\left\{\sigma_{x x}^{0}, \sigma_{\theta \theta}^{0}, 0,0,0,0\right\}$ is substituted into matrix $\mathbf{S}$, which can be written as: $\mathbf{S}=\left[\begin{array}{ccc}\sigma_{x x}^{0} \mathbf{I}_{2} & 0 & 0 \\ 0 & \sigma_{\theta \theta}^{0} \mathbf{I}_{2} & 0 \\ 0 & 0 & 0\end{array}\right]$

The integral equation can then be obtained as:

$$
\mathbf{m}_{S} \ddot{\overline{\mathbf{u}}}+\mathbf{k}_{S} \overline{\mathbf{u}}+\mathbf{f}=0
$$

where $\mathbf{f}$ is the elemental matrix of external loading, $\mathbf{m}_{S}$ is the elemental mass matrix and represented as $\mathbf{m}_{S}=$ $\int_{\Omega_{S}} \mathbf{N}_{S}^{T} \rho_{S} \mathbf{N}_{S}^{T} d \Omega_{S} ; \mathbf{k}_{S}=\mathbf{k}_{0}+\mathbf{k}_{\sigma}$ is the elemental stiffness matrix, $\mathbf{k}_{0}=\int_{\Omega_{S}} \mathbf{A}^{T} \mathbf{D A} d \Omega_{S}$ is the elemental stiffness matrix of the cylindrical shell without prestress, and $\mathbf{k}_{\sigma}=$ $\int_{\Omega_{S}} \mathbf{G}^{T} \mathbf{S G} d \Omega_{S}$ is the additional part of the elemental stiffness matrix for the cylindrical shell with prestress and defined as prestress stiffness matrix. The structural stiffness matrix was modified due to the the existence of prestress in the local area. Thus, the overall stiffness matrix was altered, the effect of fluid-structural coupling was neglected, and the mass matrix was not affected by prestress.

If the viscous damping matrix is considered, then the viscous damping matrix $\mathbf{c}_{S}$ in the differential equation can be expressed as:

$$
\mathbf{m}_{S} \ddot{\overline{\mathbf{u}}}+\mathbf{c}_{S} \dot{\overline{\mathbf{u}}}+\mathbf{k}_{S} \overline{\mathbf{u}}+\mathbf{f}=0 .
$$

The nodal displacement vector matrix was obtained by substituting the displacement vector equation and the harmonic loading equation into Eq. (12). In addition, the nodal normal velocity vector $\mathbf{v}_{n}(\omega)$ can be obtained. The nodal particle normal velocity was used as boundary condition in the acoustic radiation analysis with the boundary element method (BEM).

\section{ACOUSTIC RADIATION FUNCTION}

Structure vibration induces acoustic radiation. BEM was used to calculate the acoustic radiation behavior (acoustic pressure and acoustic radiation power) with structure response (harmonic normal velocity) as the boundary condition. Only the low-middle frequency domains of the exterior acoustic radiation of the continuum structure were analyzed in this study, which only considered the steady-state response of the vibrating structure. The standard acoustic wave equations were reduced to the Helmholtz equation in the harmonic response problem. For an arbitrary shape structure, the governing differential equation and boundary condition in steady-state linear acoustics is the classical 3D Helmholtz equation as follows:

$$
\begin{gathered}
\nabla^{2} \mathbf{p}(x, \theta, r, t)+k^{2} \mathbf{p}(x, \theta, r, t)=0 ; \\
\frac{\partial^{2} \mathbf{w}}{\partial t^{2}}=-\left.\frac{1}{\rho} \frac{\partial \mathbf{p}(x, \theta, r, t)}{\partial r}\right|_{r=R} ;
\end{gathered}
$$


where $\mathbf{p}$ is the acoustic pressure of the acoustic field point, $k$ $(=\omega / c)$ denotes the wave number, and $\omega$ and $c$ are the angular frequency and speed of sound respectively, and $\nabla^{2}$ is the Laplace operator. The acoustic wave assumes harmonic time variations throughout the analysis with $e^{i \omega t}$ dependence suppressed for simplicity.

At the structure-fluid boundaries $\Gamma_{S}$, the acoustic pressure must satisfy the Neumann boundary condition $\partial \mathbf{p} / \partial \mathbf{n}=$ $-i \omega \rho \mathbf{v}_{n}$, where $\mathbf{v}_{n}$ is the nodal normal velocity of the structure, $\rho$ is the density of the fluid medium, and $\mathbf{n}$ is the outernormal unit vector of the structure surface. Moreover, the acoustic pressure $\mathbf{p}$ precisely and automatically satisfies the Sommerfeld condition at infinity, $\lim _{r \rightarrow \infty}[\mathbf{r}(\partial \mathbf{p} / \partial \mathbf{r}-i k \mathbf{p})]=0$. The associated form of the pressure field of the cylindrical shell can be expressed by applying the variable separation method to solve the acoustic wave equation:

$$
\mathbf{p}(x, \theta, r)=\sum_{n=-\infty}^{\infty} P_{n}(x) H_{n}^{(1)}(k r) e^{i n \theta}
$$

where $n$ is the expansion coefficient, $H_{n}^{(1)}(k r)$ is the $n$-th order Hankel function of the first kind, $P_{n}(x)$ is the pressure amplitude, and $k=\sqrt{k_{f}^{2}-k_{x}^{2}}, k_{f}=\omega / c$. In addition, the radial velocity $\mathbf{v}_{n}(\vec{r})$ in the acoustic field is $\mathbf{v}_{n}(\vec{r})=-\frac{1}{i \omega \rho} \frac{\partial \mathbf{p}}{\partial \mathbf{r}}$.

In this study, the acoustic medium was defined as air. The feedback coupling between the acoustic medium and the vibrating structure was also neglected because of its weak influence. The acoustic radiation field quantities were relevant to acoustic radiation pressure and power. However, acoustic pressure varies according to spatial position, and the calculation is time consuming. Acoustic power was a suitable parameter to be used for quantifying the radiation on the structure surface. Acoustic power is also related to the characteristics of structure vibration and does not change with spatial position. Therefore, the power parameter of acoustic radiation is preferred over the acoustic pressure parameter for structuralacoustic analysis and evaluation. In the acoustic field domain, the acoustic radiation power describes the energy flow of an assumed integral surface and can be defined as:

$$
\Pi=\frac{1}{2} \int_{\Gamma} \operatorname{Re}\left\{\mathbf{p}(\vec{r}) \cdot \mathbf{v}_{n}^{*}(\vec{r})\right\} d \Gamma ;
$$

where "Re" represents the real part of a complex variable, $\mathbf{p}(\vec{r})$ is the acoustic pressure at the acoustic field point, as shown in Eq. (15), $\mathbf{v}_{n}^{*}(\vec{r})$ is the field-point normal complex conjugate operator velocity of fluid-particle, and $\Gamma$ is the integral surface of acoustic domain. After omitting the acoustic transmission loss and absorption of the boundary and source points, the acoustic radiation power of the exterior acoustic field is equal to that of the source point surface. The structure surface radiation acoustic power can be written as:

$$
\Pi=\frac{1}{2} \operatorname{Re} \int_{\Gamma} \mathbf{p}_{f} \mathbf{v}_{n}^{*} d \Gamma ;
$$

where $\Gamma$ is the structure-fluid interface, $\mathbf{p}_{f}$ is the acoustic pressure on the structural surface, and $\mathbf{v}_{n}^{*}$ is the nodal normal complex conjugate velocity on the structural surface. In the vibration structure, $\mathbf{p}_{f}=\rho c \mathbf{v}_{n}$ exits, where the phase angle between acoustic pressure $\mathbf{p}_{f}$ and the normal velocity $\mathbf{v}_{n}^{*}$ is 0 .

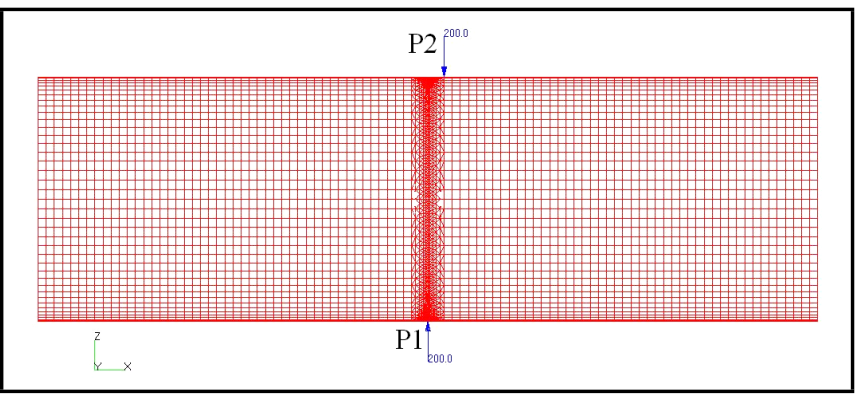

Figure 3. Finite element model of the cylindrical shell.

\section{NUMERICAL RESULTS AND DISCUSSION}

A cylindrical shell structure under external harmonic excitation in air was used to demonstrate the influence of prestress in local areas on the structural-acoustic characteristics of the shells. In the numerical examples, FEM was used to analyze structural dynamic response, whereas BEM was applied to deal with the exterior acoustic radiation problem, structure vibration, acoustic power, and acoustic directivity. This study only focused on the steady-state dynamic response of the vibrating structure.

\subsection{Model Description}

A finite thin cylindrical shell structure with supported boundary condition immersed in fluid was used in this study, as shown in Fig. 3. The radius, length, and thickness of the cylindrical shell are $1.5,4.8$, and $0.01 \mathrm{~m}$ respectively. The cylindrical shell structure was discretized using FEM. In the model, the zero point of the overall coordinate system is located at the center of the cylindrical shell.

The material of the cylindrical shell structure is steel with mechanical performance parameters as follows: density, $\rho=$ $7800 \mathrm{~kg} / \mathrm{m}^{3}$; modulus of elasticity, $E=210 \mathrm{GPa}$; and the Poisson ratio, $\mu=0.3$. The total weight of the cylindrical shell is $1764.0 \mathrm{~kg}$, whereas the fundamental frequency is $\omega=$ $7.6 \mathrm{~Hz}$.

In the structural-acoustic analysis, the acoustic properties of the fluid (i.e. air) are isotropic and homogeneous, with density $(\rho)$ of $1.225 \mathrm{~kg} / \mathrm{m}^{3}$, speed of sound $(c)$ of $343 \mathrm{~m} / \mathrm{s}$, acoustic power reference value $\left(W_{0}\right)$ of $1 \times 10^{-12}$ Watt, and acoustic pressure reference value $\left(p_{0}\right)$ of $2 \times 10^{-5} \mathrm{~Pa}$.

A time-harmonic line force loading on the shell structure was also observed. The first concentrated loading is $\mathbf{f}(t)=F \sin (2 \pi f t)$, with a prescribed amplitude of $P<$ $0,0,200.0>\mathrm{N}$. The frequency of the external force is $f=$ $61 \mathrm{~Hz}$, which was applied to a point on P1, as shown in Fig. 3. The second concentrated loading is $\mathbf{f}(t)=F \sin (2 \pi f t)$, with a prescribed amplitude of $P<0,0,-200.0>\mathrm{N}$. The frequency of the external force is $f=61 \mathrm{~Hz}$, which was applied to a point on $\mathrm{P} 2$, as shown in Fig. 3.

\subsection{Structure Prestress}

Prestress of the cylindrical shell structure was induced by ring static loading. A uniform distribution of static loading was observed on the cylindrical shell structure, as shown in Fig. 3. The length of the static loading region is $0.2 \mathrm{~m}$ on the axis direction. The amplitude of the static surface force is $1.6 \times 10^{6} \mathrm{~Pa}$, and the normal direction of the force is inward.

The deformation parameter of the cylindrical shell structure was obtained according to the numerical analysis of the structure subjected to static loading. In addition, the contour maps 


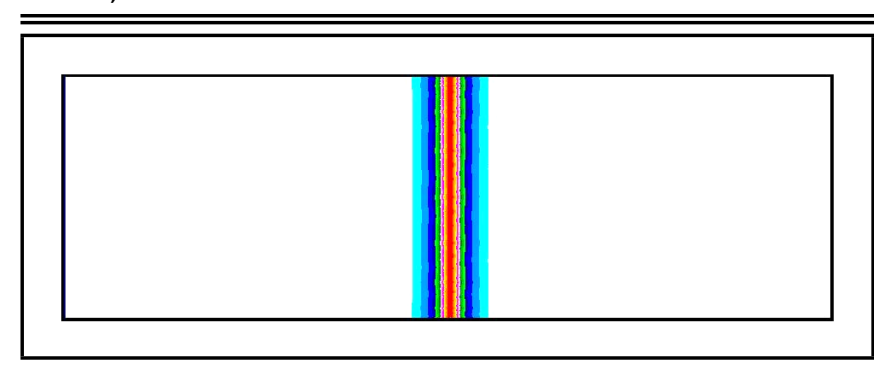

Figure 4. Contour maps of prestress distribution.

Table 1. Comparison of mode frequencies.

\begin{tabular}{|c|c|c|}
\hline Mode order & Without prestress & With prestress \\
\hline 1 & 7.6 & 7.2 \\
2 & 9.1 & 8.9 \\
3 & 12.0 & 11.0 \\
4 & 20.0 & 19.4 \\
5 & 20.1 & 20.0 \\
6 & 33.8 & 32.5 \\
\hline
\end{tabular}

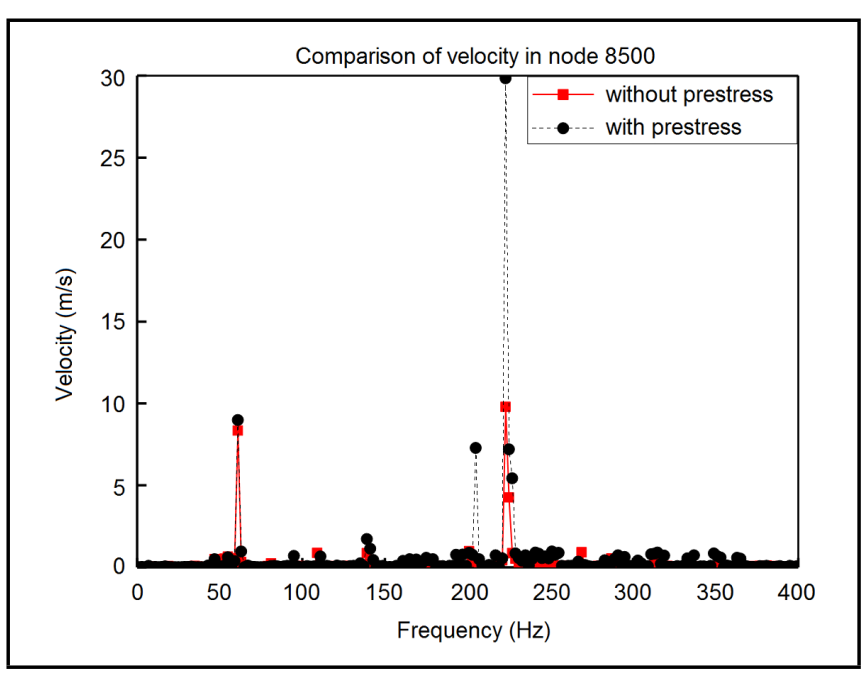

Figure 5. Comparison of velocity.

of the structure stress distribution are illustrated in Fig. 4. The structure stress is concentrated in the center of the shell structure near the static loading region. Far from the static loading region, the stress is very small and can be omitted. The maximum value of the structure stress of the shell structure is $107 \mathrm{MPa}$. The structure stress is defined as local-area prestress in the dynamic response analysis.

\subsection{Vibration of the Cylindrical Shell}

The dynamic characteristic of the cylindrical shell structure changed with prestress distribution. Table 1 shows the mode frequencies of the structure with or without prestress distribution. The influence of prestress on the dynamic behavior of the shell structure is considered significant.

The vibration characteristics of the cylindrical shell were compared in the absence or presence of prestress distribution. The velocity of the cylindrical shell structure can be calculated using the FEM code. Frequency variation in the analysis ranges from $0 \mathrm{~Hz}$ to $400 \mathrm{~Hz}$, and the frequency step size is $2 \mathrm{~Hz}$. Figure 5 demonstrates the comparison of the node velocity of the defined point located on the static loading region with or without prestress.

As shown in Fig. 5, the influence of prestress on the dynamic behavior of the cylindrical shell structure is evident.

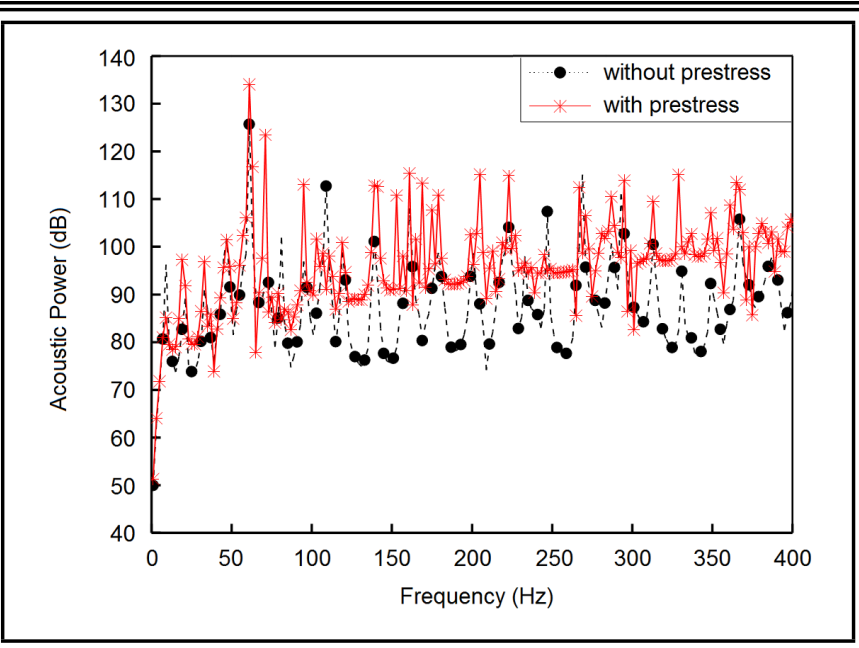

Figure 6. Comparison of acoustic power.

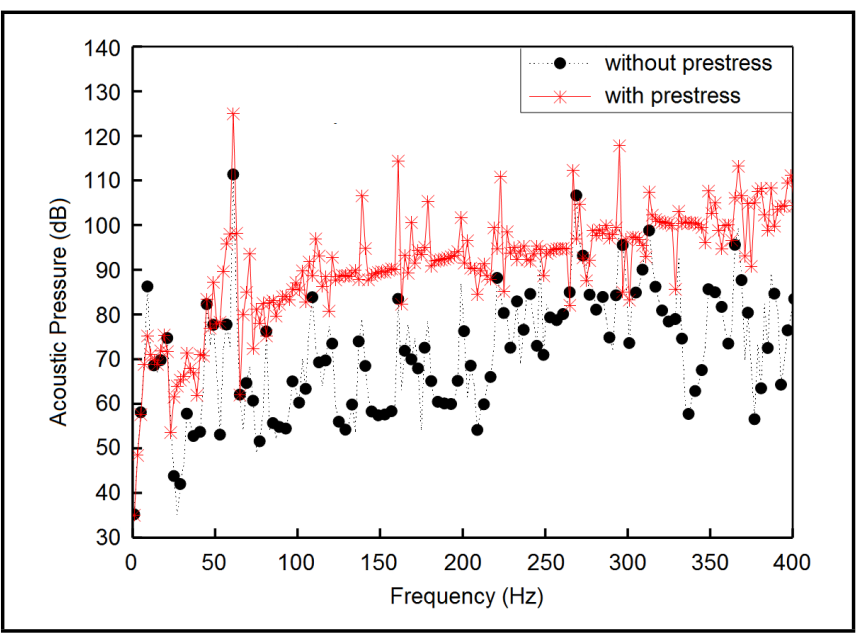

Figure 7. Comparison of acoustic pressure.

\subsection{Acoustic Radiation of the Cylindrical Shell}

Acoustic pressure in the field point and acoustic radiation power were obtained using the BEM code combined with the normal velocity of the cylindrical shell structure. Frequency variation in radiated acoustic power ranges from $0 \mathrm{~Hz}$ to $400 \mathrm{~Hz}$, and the frequency step size is $2 \mathrm{~Hz}$. Figure 6 shows the comparison between the acoustic radiation power with and without prestress. As shown in Figs. 6 and 7, the influence of prestress on acoustic radiation power, as well as on acoustic pressure in the field point of the cylindrical shell structure is evident, with the field point located in $(0,5$, and 5$)$.

Numerical analysis showed that the stiffness matrix of the cylindrical shell structure and the dynamic response parameter were altered in the presence of prestress. Acoustic radiation power was then changed, and the maximum value of the acoustic radiation power significantly increased.

\subsection{Acoustic Directivity Analysis}

The influence of prestress, which is located in local areas of the acoustic source point on acoustic pressure directivity was compared. In the directivity analysis, acoustic pressure was compared in the frequency of $61 \mathrm{~Hz}$. The acoustic field was located in the horizontal plane midway along the cylinder length. Figure 8 shows the comparison between the acoustic radiation directivity with and without prestress. 


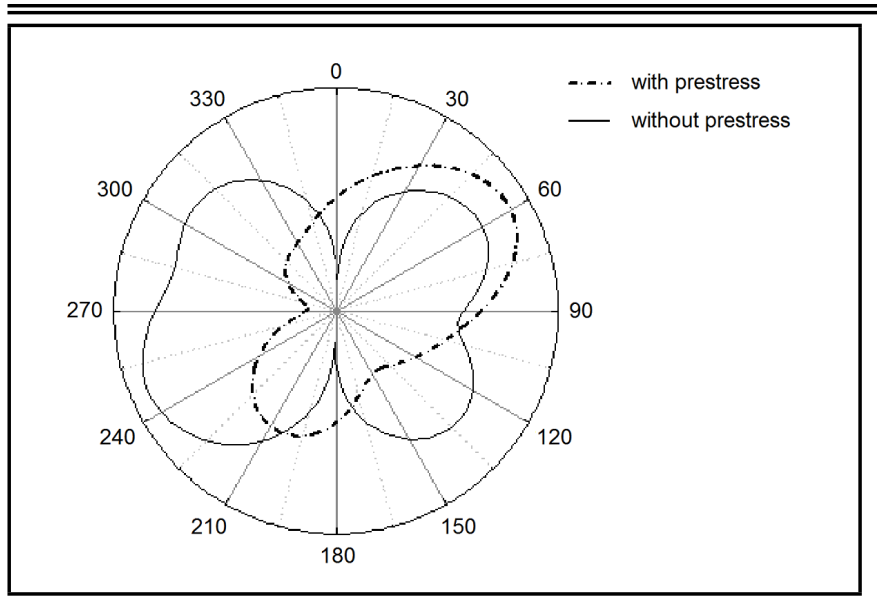

Figure 8. Comparison of the acoustic directivity.

A comparison of Figs. 5, 6, 7, and 8 shows that the dynamic characteristics are significantly reduced. Additionally, the influence of the existing prestress is obvious.

\section{CONCLUSIONS}

In this study, acoustic radiation power is proposed as a performance index to address vibration and acoustic radiation problem of a prestressed cylindrical shell structure in local area distribution. The following conclusions are drawn through numerical analysis and comparison. The existence of local prestress changes the stiffness of the structure. Prestress distributed in local areas significantly influences sound radiation and is evident in low-frequency band. Meanwhile, the structure of radiation directivity can be changed. Future studies will focus on fluid-structure coupling characteristics at the interface.

\section{FUNDING}

This work was partially supported by the State Key Laboratory of Ocean Engineering of Shanghai Jiaotong University. Their support is gratefully acknowledged by the authors.

\section{REFERENCES}

1 Nayak, A. K., Moy, S. S. J., and Shenoi, R. A. A higher order finite element theory for buckling and vibration analysis of initially stressed composite sandwich plates, Journal of Sound and Vibration, 286, 763-780, (2005). http://dx.doi.org/10.1016/j.jsv.2004.10.055

2 Saiidi, M., Douglas, B., and Feng, S. Prestress force effect on vibration frequency of concrete bridges, Journal of Structural Engineering, 120, 22332241, (1994). http://dx.doi.org/10.1061/(asce)07339445(1994)120:7(2233)

3 Naguleswaran, S. Transverse vibration of an uniform Euler-Bernoulli beam under linearly varying axial force, Journal of Sound and Vibration, 275, 47-57, (2004). http://dx.doi.org/10.1016/s0022-460x(03)00741-7

4 Haddadpour, H., Mahmoudkhani, S., and Navazi, H. M. Free vibration analysis of functionally graded cylindrical shells including thermal effects, Thin-Walled Structures, 45, 591-599, (2007). http://dx.doi.org/10.1016/j.tws.2007.04.007
5 Hamed, E. and Frostig, Y. Natural frequencies of bonded and unbonded prestressed beams prestress force effects, Journal of Sound and Vibration, 295, 28-39, (2006). http://dx.doi.org/10.1016/j.jsv.2005.11.032

6 Bigoni, D., Gei, M., and Movchan, A. B. Dynamics of a prestressed stiff layer on an elastic half space: filtering and band gap characteristics of periodic structural models derived from long-wave asymptotics, Journal of the Mechanics and Physics of Solids, 56, 2494-2520, (2008). http://dx.doi.org/10.1016/j.jmps.2008.02.007

7 Lundqvist, P. and Rydén, N. Acoustoelastic effects on the resonance frequencies of prestressed concrete beams-shortterm measurements, NDT \& E International, 50, 36-41, (2012). http://dx.doi.org/10.1016/j.ndteint.2012.04.010

8 Zhou, H. J., Li, W. Y., Lv, B. L., et al. Free vibrations of cylindrical shells with elastic-support boundary conditions, Applied Acoustics, 73, 751-756, (2012). http://dx.doi.org/10.1016/j.apacoust.2012.02.008

9 Zhu, X., Ye, W. B., and Li, T. Y. The elastic critical pressure prediction of submerged cylindrical shell using wave propagation method, Ocean Engineering, 58, 22-26, (2013). http://dx.doi.org/10.1016/j.oceaneng.2012.09.008

10 Zhang, Y. L., Daniel, G. G., and Jason, M. R. Vibration of prestressed thin cylindrical shells conveying fluid, Thin-Walled Structures, 41, 1103-1127, (2003). http://dx.doi.org/10.1016/s0263-8231(03)00108-3

11 Zhang, Y. L., Reese, J. M., and Gorman, D. G. Finite element analysis of the vibratory characteristics of cylindrical shells conveying fluid, Computer methods in applied mechanics and engineering, 191, 5207-5231, (2002). http://dx.doi.org/10.1016/s0045-7825(02)00456-5

12 Bakhtiari-Nejad, F. and Bideleh, S. M. M. Nonlinear free vibration analysis of prestressed circular cylindrical shells on the Winkler/Pasternak foundation, Thin-Walled Structures, 53, 26-39, (2012). http://dx.doi.org/10.1016/j.tws.2011.12.015

13 Amabili, M. A. comparison of shell theories for largeamplitude vibrations of circular cylindrical shells: Lagrangian approach, Journal of Sound and Vibration, 264, 1091-1125, (2003). http://dx.doi.org/10.1016/s0022$460 x(02) 01385-8$

14 Zhou, X. P. Vibration and stability of ring-stiffened thin-walled cylindrical shells conveying fluid, Acta Mechanica Solida Sinica, 25, 168-176, (2012). http://dx.doi.org/10.1016/s0894-9166(12)60017-2

15 Belyankova, T. I. and Kalinchuk, V. V. The dynamic contact problem for a prestressed cylindrical tube filled with a fluid, Journal of Applied Mathematics and Mechanics, 73, 209-219, (2009). http://dx.doi.org/10.1016/j.jappmathmech.2009.04.011

$16 \mathrm{Hu}$, X. J. and Redekop, D. Prestressed vibration analysis of a cylindrical shell with an oblique end, Journal of Sound and Vibration, 277, 429-435, (2004). http://dx.doi.org/10.1016/j.jsv.2003.11.051 